\title{
Large-scale Text-based Video Classification using Contextual Features
}

\author{
Zein Al Abidin Ibrahim, Siba Haidar, and Ihab Sbeity
}

\begin{abstract}
The production of video has increased and expanded dramatically. There is a need to reach accurate video classification. In our work, we use deep learning as a mean to accelerate the video retrieval task by classifying them into categories. We classify a video depending on the text extracted from it. We trained our model using fastText, a library for efficient text classification and representation learning, and tested our model on 15000 videos. Experimental results show that our approach is efficient and has good performance. Our technique can be used on huge datasets. It produces a model that can be used to classify any video into a specific category very quickly.
\end{abstract}

Index Terms-Deep learning, Text-based video classification, Contextual information, fastText.

\section{INTRODUCTION}

The amount of video archives that we have are increasing tremendously day by day. Due to the ease of use of internet and latest technologies are making it easy to share videos. With a simple click on any smartphone, a video is created and shared with the whole world and on different Social Media platforms such as Facebook, YouTube, WhatsApp, Instagram and many others. This is leading to lots of duplication too. Finding out the type of videos you want to see is a very difficult task. Such a time consuming and tedious job must be made automatic. This task is called video classification.

The searching that we have done showed that the analysis of the audiovisual content is the main step for better storage solutions, video recommendation systems, profile construction, duplication detection (for rights issues) and many other systems. For example, knowing the type of videos shared and viewed by a user may constitute information about his interests which will later facilitate the task of any recommendation system. In this work, we address the above shortcoming by finding the most suitable deep learning technique helping in text-based video classification.

It is now clear that the production of videos has become as simple as pressing a button. However, it rarely happens that a shared video has information that describes well its content and categorizes it, which makes extremely hard for us when we try to search for a certain video under a specific category. For a video to be categorized without any intrusion, it would depend on hashtags that have already been classified, that is if the tags are available, or by analyzing some of the keywords present in the title of the video. Using this technique will result in high misclassification rate in most of the cases since they are relying on inaccurate data.

Automatic video classification is the process of generating

Z..I.: Zein Al Abidin Ibrahim, Associate professor at the Lebanese University- Faculty of Sciences - Section 1, Beirut, Lebanon (e-mail: zein.ibrahim@ul.edu.lb). relevant labels to a video or to different parts of video automatically without human intervention. The video content should be extracted and understood to find the type of video it belongs to. It is a main step for better storage solutions, video recommendation systems, profile construction, duplication detection (for rights issues) and many other systems.

\section{VIDEO CLASSIFICATION TECHNIQUES}

Classification process, in general, requires features based on which one can distinguish among the categories. These features are mainly taken from text, audio or visual content of the video. Based on that, there are three types of classification techniques.

\section{A. Video-based Classification}

Most of the researchers have used this type of methods since most of the content are perceived visually by humans. Considering the video as a collection of frames, visual features are mostly color-based, motion-based, and/or objectbased features. In this latter approach, first, the objects are identified and then some of their features are extracted. For example, faces can be detected in video and then features like skin tone, size, and position are extracted from faces.

\section{B. Audio-based Classification}

The audio modality is the second most used type of modalities for video classification. Storage of audio and its features requires less space than the video and text. As Mittal C. Darji [1] mentioned, we have two types: the physical features which are low-level features directly measured from frequency values of the signal, and the perceptual features which are audio features containing variation in values based on the category of audio. For example, music has higher continuous amplitude than speech. These kinds of observations are used by researchers to classify videos.

\section{Text-based Classification}

In this category, text is produced from video and then analyzed for classification. Text can be either visible on the screen, or extracted from the speech. In the former case, the text visible on screen is extracted. For example, the scoreboard of game, number on jersey of player, captions are shown on the screen etc. Such text can be extracted using Optical Character Recognition (OCR). In the latter case, the text is extracted from speech using speech recognition.

S. H. Siba Haidar, Associate professor at the Lebanese UniversityFaculty of Sciences - Section 1, Beirut, Lebanon (e-mail: siba.haidar@ ul.edu.lb)

I. S. Ihab Sbeity, professor at the Lebanese University- Faculty of Sciences - Section 1, Beirut, Lebanon (e-mail: ihab.sbeity@ ul.edu.lb) 
Textual information is rich of information helping in identifying some clues about video categories. In our approach, we will base on the text extracted from the speech transcripts. The paper is organized as follows: Section III introduces the basics of text classification and clarifies some of its tools and techniques. Section IV reviews some of the methods in the video classification domain. In Section V, we introduce the dataset and the different scenarios we used during our experimentations. Section VI presents the conclusion and the future works.

Table 1: NLP Tasks

\begin{tabular}{lll}
\hline \multicolumn{1}{c}{ Table 1: NLP Tasks } & \\
\hline \multicolumn{1}{c}{ Easy Task } & \multicolumn{1}{c}{ Medium Task } & \multicolumn{1}{c}{ Hard Task } \\
\hline Chunking & Syntactic Parsing & Machine Translation \\
Part-of-Speech & Word Sense & Text Generation \\
Tagging & Disambiguation & Automatic \\
Named Entity & Sentiment Analysis & Summarization \\
Recognition & Topic Modeling & Question Answering \\
Spam Detection & Information Retrieval & Interfaces \\
Thesaurus & & \\
\hline \hline
\end{tabular}

\section{TEXT ClASSIFICATION}

\section{A. NLP}

NLP is a sub-field of Artificial Intelligence (AI) aiming to build intelligent computers that can behave like a human being [2]. It is the study of interaction between computers and human languages. One of the important applications of NLP (see Table 1) is: Speech-to-Text, figure 1.

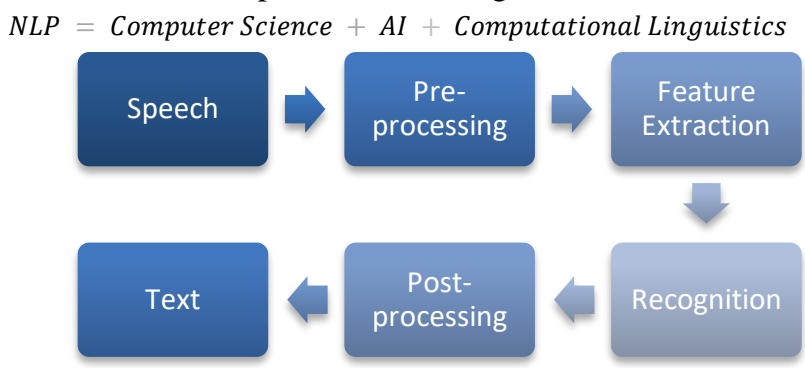

Fig. 1. Speech-to-Text (right to left)

We have several terminologies of NLP. Two of the main terminologies are Machine Learning and Text Mining. Machine learning is a subfield of computer science (CS) and artificial intelligence (AI). There are several applications of machine learning such as spam email filtering, objects detection, and optical character recognition (OCR). We have various machine learning tasks and in many forms. In supervised learning, we have a goal which is to learn the rules that map inputs to outputs. Supervised learning is applied for example in spam email filtering aiming to specify whether an email is considered as spam or not. On the other hand, unsupervised learning is used to discover hidden patterns in data where the labels are not given to the learning algorithm. Topic modeling is one of the applications of unsupervised learning where the program is given a group of documents and it must classify them into several topics.

Text mining is one form of application of data mining. Data mining is a process that takes data as input and outputs knowledge. Text Mining tasks consist of:

1) Text Clustering: Finding groups of objects such that the objects in a group are similar (or related) to one another and different from (or unrelated to) the objects in other groups.

2) Text Summarization: Reducing the size of inputted text in order to create a summary that returns the most important points of the original text.

3) Text Classification: categorizing a large document which is defined as a process of assigning unlabeled documents to pre-described classes automatically.

Some of the traditional and effective techniques used in text classification is TF*IDF. It is a technique used to retrieve information that measures a term's frequency (TF) and its inverse document frequency (IDF). Each word has its own TF and IDF score. Their product is called the TF*IDF weight.

Another widely used method for text classification is the Singular Value Decomposition (SVD). It is an unsupervised learning algorithm, which is used by global companies such as Google, Netflix, Facebook, YouTube, and others[3]. Other techniques exist like Co-Occurrence Matrix, Count-based and Predictive models.

\section{B. Text Classification Techniques}

Latent semantic analysis (LSA) is a technique in natural language processing, used to analyze relationships between a set of documents and the terms they contain. LSA expects that words that are close in meaning will occur in similar pieces of text. A matrix containing word counts is created from a large piece of text and then SVD is applied on it to reduce the number of rows. Words are then compared by taking the cosine of the angle between the two vectors formed by any two rows. Values close to 1 represent very similar words while values close to 0 represent very dissimilar words.

GloVe is a "count-based" model based on word occurrences in a corpus. It is an unsupervised learning algorithm used to obtain vector representations for words [4]. Training is done using word-word co-occurrence matrix. In fact, it is based on two main steps: The first one is the construction of a co-occurrence matrix $\mathrm{X}$ from a training set. The second step is the factorization of $\mathrm{X}$ in order to get vectors.

Word2Vec is a "predictive" model based on word embeddings. Word2Vec models help derive relations between a word and its context [5] using a two-layer neural network. Word2Vec predicts words based on their context by using one of two neural models: CBOW or Skip-Gram.

\section{FastText}

With the growth of data, we need to have more important tools to better understand the content of large datasets in order to achieve more accurate results. [6]

For this need, the Facebook AI Research (FAIR) lab is opensourcing fastText, a library designed to help build scalable solutions for text representation and classification. FastText combines some of the most successful concepts introduced by the natural language processing and machine learning communities in the last few decades. One of the concepts of fastText is to represent sentences with the bag of words and bag of n-grams. We can train fastText on more than one billion words in less than ten minutes using a standard multicore CPU, and classify half a million of sentences in $312 \mathrm{~K}$ classes in less than a minute. 


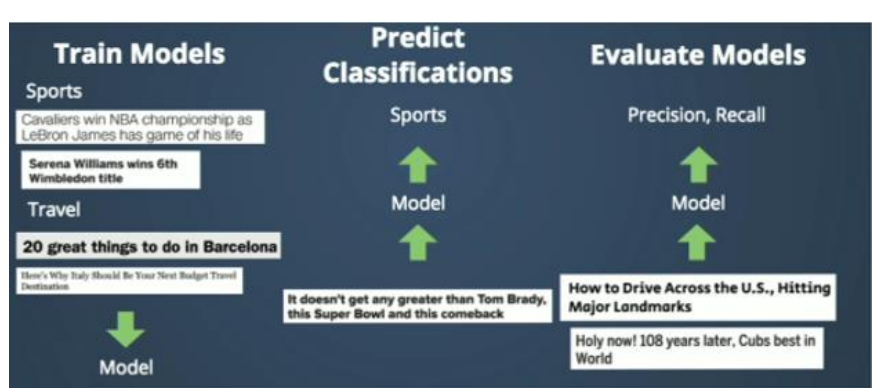

Fig. 2. FastText functionality

FastText differs from word2vec only in that it uses char ngram embedding as well as the actual word embedding in the scoring function. Also, fast Text contains most of the concepts in natural language processing and machine learning like representing sentences with a bag of words and bag of ngrams, as well as using sub-word information, and sharing information across classes through a hidden representation. These different concepts are being used for two different tasks: efficient text classification and learning word vector representations.

\section{RELATED WORK}

When users upload videos to Web sites, they usually specify category labels with them and may provide some textual description about the content. Labeling a video is not a trivial task as someone may think. Users may suffer to choose an appropriate category for a video. Two different users may provide two different labels for the same video because they have different understandings about video categories. Thus, the user labeled categories may often be inconsistent [7].

The semantics of videos are described by both audio-visual content features and textual descriptions. The previous work on automatic video classification by [8] exploits both text features and audio-visual content features for video categorization, and the results show that text features consistently outperform video content features. However, the performance of using both text features and video content features is better than the performance of using either of them alone.

X. Yang in [9] treats the text description of a video as a bag of words to build classifier as in text classification for general documents. There are some problems with the text descriptions of videos such as that they are quite short. Additionally, the descriptions of different videos usually share very few identical words. That is, the feature space is extremely rare. Another problem is that the text descriptions of Web videos often contain special words and abbreviations, such as names of persons or organizations (e.g. "TNA" for "Total Nonstop Action").

$\mathrm{Wu}$ et al. [10] proposed the integration of social information for video classification in a similar setting. The social and contextual information they use include related videos and user upload habits. Classification is done by combining scores from an SVM classifier trained using videos' text metadata, and confidences computed using the sets of related videos and user's uploaded videos. Evaluation is performed using over 6,000 videos across 15 categories. Lin et al. [11] combine text feature from captions and visual features from the video clips and use Support Vector Machine
(SVM) classifier.

Early works on video classification were based on Hidden Markov Models (HMM). Audio and visual features were integrated and fed into two-stage HMM. A new HMM was built for each new feature discovered. The concatenation approach gives better performance only if the features are highly correlated. Face and text trajectories were extracted from video clips and an HMM was used to classify a given video clip into some predefined categories.

\section{PROPOSED METHOD}

In this article, we propose to use fastText for video classification. The text is the output of a speech transcription tool applied on the audio signal of the videos. Several scenarios have been applied. A main scenario is to show how much a model trained on separate dataset is efficient to classify another dataset in comparison with a model trained and tested on the same dataset.

\section{EXPERIMENTATION}

Text classification is an area where classification algorithms are applied on a piece of text. The task is to assign a text into one (or more) classes, based on its content. Typically, these classes are chosen by humans. Some of the popular areas where text classification is applied are as follows: classify news as Politics, Sports, World, Business, Lifestyle, email as Spam, Other, etc.

\section{A. Hyperparameters used by FastText}

By default, fastText sees each training example only five times during training, which is pretty small. The number of times each example is seen (also known as the number of epochs), can be increased using the -epoch option. Learning rate is a hyper-parameter that controls how much we are adjusting the weights of our network with respect to the loss gradient. Another way to change the learning speed of our model is to increase (or decrease) the learning rate of the algorithm. This corresponds to how much the model changes after processing each example. A learning rate of 0 would mean that the model does not change at all, and thus, does not learn anything. Good values of the learning rate are in the range $[0.1-1.0]$.

We can improve the performance of a model by using word bigrams, instead of just unigrams. This is especially important for classification problems where words' order is important, such as sentiment analysis. A 'unigram' refers to a single atomic unit, or token, usually used as an input to a model. In fastText, we work at the word level and thus unigrams are words. Similarly, we denote by 'bigram' the concatenation of 2 consecutive tokens or words. We often talk about n-gram to refer to the concatenation of any $n$ consecutive tokens. For example, in the sentence, 'Last donut of the night', the unigrams are 'last', 'donut', 'of', 'the' and 'night'. The bigrams are: 'Last donut', 'donut of', 'of the' and 'the night'. Bigrams are particularly interesting because, for most sentences, you can reconstruct the order of the words just by looking at a bag of n-grams.

On the other hand, since we are training our model on a few thousands of examples, the training only takes a few seconds. But training models on larger datasets, with more 
labels can start to be too slow. A potential solution to make the training faster is to use the hierarchical softmax, instead of the regular softmax.

\section{B. Our DataSet}

The dataset used during our experimentations is the Blip10000 dataset, which consists of 14,838 videos for a total of 3,288 hours from blip.tv [8]. This dataset was designed for tagging and retrieval tasks; Videos are split into 2 sets: DevSet contains 5,288 videos used for training our model and TestSet contains 9,550 videos used for testing our model. We have 22 categories. Data is stored in UTF-8-formatted XML files for each video including information about the speech transcripts. They were extracted from the audio streams that were preprocessed using a combination of ffmpeg and sox software. Most of videos are English spoken, but there are also some of them in Czech, Dutch, French, Italian, German and Spanish. Among the categories, we find the default category that contains all the videos that were not specified a category when uploaded by the user and all the categories that contains less than 100 videos. Thus, the default category contains videos of different types.

After filtering the two datasets by removing silent videos (no speech) and the non-English ones from the 2 sets, the number of videos remaining are as follows; In the DevSet, the total number of videos without Default-Category is 3667 . The total number of videos with Default-Category 4214. As for the TestSet, the total number of videos without DefaultCategory 4175 . The total number of videos with DefaultCategory 4945.

Let us note that in all our work we used python language for training, testing and for cleaning our videos. The distribution of videos in the two sets among the categories are shown in table 2.

\section{Scenarios}

During our experimentations, we have tested different scenarios as presented in the next subsections. The training phase based on a vocabulary website [12] that gave us all the related words to each word given to it and/or the speech transcripts of some videos in our dataset. Please refer to table 3 for information on each scenario settings.

Scenario 1: we have started our testing by considering only 4 videos per category chosen randomly for testing the model. We have started by using two categories only (Food \& Vehicles as shown in figure 3), then we started to increase the number of used categories (figures 4, 5 and 6). All the words for training our model are extracted from the above reference [12]. By classifying 4 random videos from each of the 21 categories (excluding the Default-Category), we have reached a classification accuracy of $47 \%$.

Scenario 2: In this scenario, we have trained our model using [12] and tested it on the whole DevSet of [8]. The accuracy was too low $(6 \%)$ which may due to the fact that the trained model is not good enough for the videos we are using in the test phase.

Scenario 3: We divided our DevSet into 2 groups: one for training and one for testing after excluding the default category. Here, we used the first group of videos to train our model and the second group for testing. The classification accuracy increased up to $60 \%$.

Table 2: DATASET DISTRIBUTION OVER CATEGORIES

\begin{tabular}{ccc}
\hline \hline Easy Task & DevSet & TestSet \\
\hline Autobiography & 47 & 56 \\
Business & 84 & 95 \\
Comedy & 151 & 225 \\
Conference & 68 & 82 \\
Documentary & 61 & 131 \\
Education & 319 & 512 \\
Environment & 45 & 98 \\
Food & 83 & 117 \\
Gaming & 98 & 171 \\
Health & 88 & 157 \\
Journalism & 116 & 158 \\
Literature & 83 & 109 \\
Media & 105 & 164 \\
Movies & 263 & 366 \\
Music & 194 & 328 \\
Politics & 595 & 278 \\
Religion & 259 & 351 \\
Sports & 179 & 232 \\
Technology & 779 & 461 \\
Travel & 43 & 73 \\
Vehicles & 7 & 11 \\
Default-Category & 547 & 770 \\
\hline \hline
\end{tabular}

Table 3: SCENARIOS

\begin{tabular}{|c|c|c|c|c|c|c|}
\hline 象 & 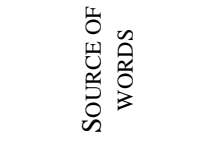 & 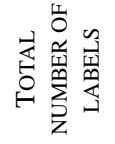 & 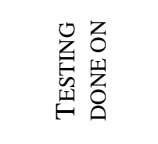 & 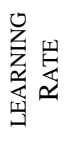 & 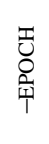 & 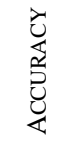 \\
\hline 1 & [12] & 21 & 4 / category & 1.0 & 80 & $47 \%$ \\
\hline 2 & [12] & 21 & all DevSet & 1.0 & 50 & $6 \%$ \\
\hline 3 & {$[12]+$ half DevSet } & 21 & half DevSet & 1.0 & 50 & $60 \%$ \\
\hline 4 & {$[12]+$ half DevSet } & 22 & half DevSet & 1.0 & 50 & $35 \%$ \\
\hline 5 & [12]+ DevSet & 21 & from TestSet & 1.0 & 50 & $36 \%$ \\
\hline 6 & [12]+ DevSet & 22 & from TestSet & 1.0 & 50 & $23 \%$ \\
\hline 7 & DevSet & 22 & TestSet & 1.0 & 50 & $36 \%$ \\
\hline
\end{tabular}

Scenario 4: This scenario is similar to the previous one. The only difference is that we have included the DefaultCategory. The accuracy dropped down to $35 \%$.

Scenario 5: In scenario 5, we have again excluded the Default-Category from the training and testing phases. The training was done using [12] and the DevSet and the testing on TestSet (excluding the Default-Category). The accuracy obtained is $36 \%$.

Scenario 6: Similar to the previous scenario but we have considered here the Default-Category. The accuracy decreased to $23 \%$.

Finally, in scenario 7, we have trained our model on the whole DevSet and tested it on the whole TestSet. The accuracy obtained is $36 \%$.

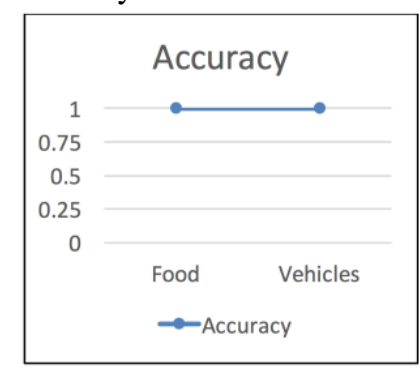

Fig. 3. Results Using 2 Categories

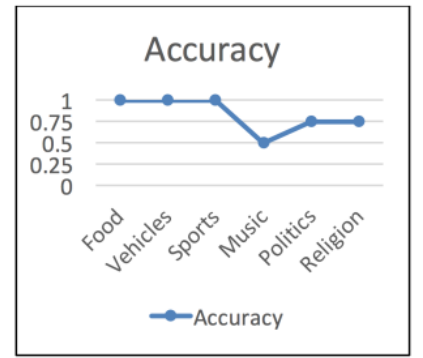

Fig. 4. Results Using 6 Categories 


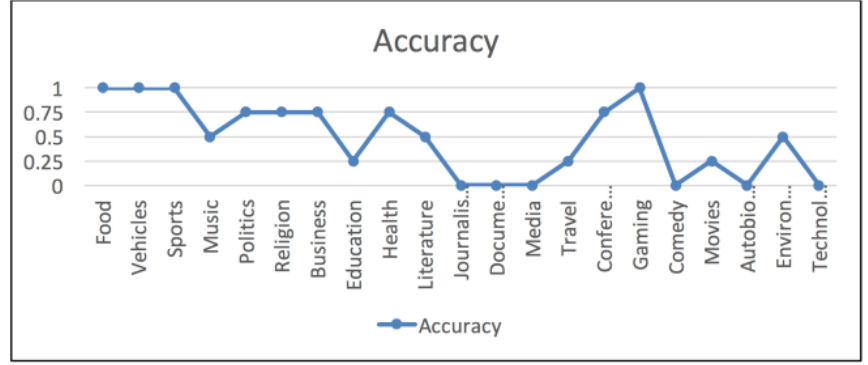

Fig. 6. Results Using 21 Categories

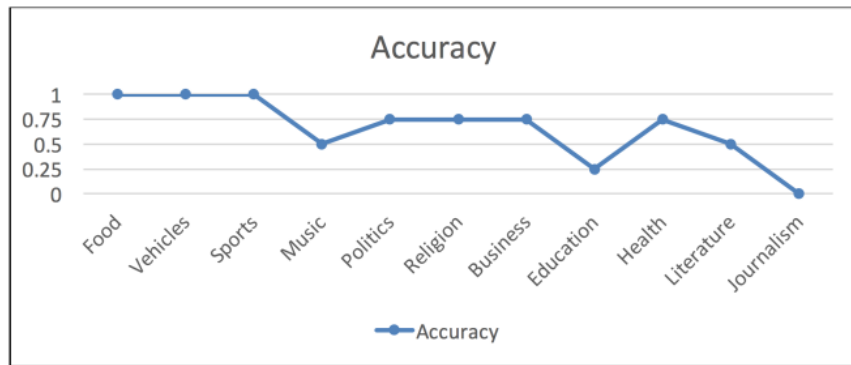

Fig. 5. Results Using 11 Categories

\section{DISCUSSION}

If we consider the scenario 7 which is the most logical one, the accuracy obtained was $36 \%$. This percentage doesn't reflect the accuracy of all the categories. In other words, some categories reached an accuracy of $78 \%$ and $70 \%$, but other categories got low percentages.

In order to judge the efficiency of our work, we have compared it to six works that have participated to the tagging task on the same dataset ARF [13], KIT[14], TUB[15], TUD[16], TUD_MM[17], UNI_Camp[18]. In these works, one or more modalities have been considered for the classification. Table 4 shows the obtained results on the same dataset.

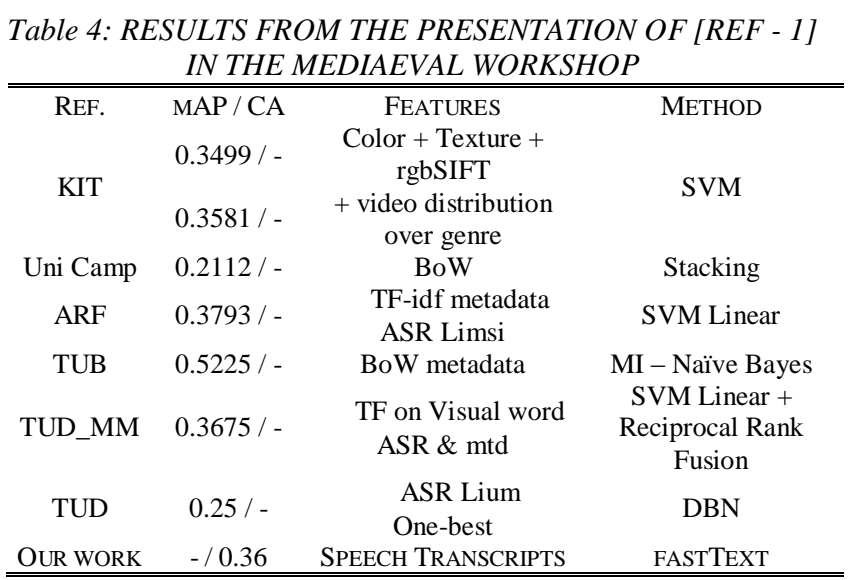

As shown in the table 4, the obtained results are competitive to the results of the shown works. We had no chance to compare our work to deep-learning video classification approaches since we do not have the same dataset. Even for the works that the datasets are available such as youtube $1 \mathrm{M}$ and youtube $8 \mathrm{M}$, these datasets do not provide the raw videos but only features extracted from some frames inside the videos.

\section{CONCLUSION}

In this article, we have proposed a text-based video classification technique of unstructured videos. The text is derived from the speech transcripts. In order to take into account context information of text, we have used the fastText method developed by Facebook AI team. The experimentations done on a 15000 unstructured videos dataset showed a competitive accuracy to the works of the literature tested on the same dataset. In the near future work, we aim to integrate several modalities in the classification in order to enhance it.

\section{REFERENCES}

[1] M. Darji and D. Mathpal, "A review of video classification techniques," IRJET Journal, vol. 4. no. 6, June 2017.

[2] G. Kaur and P. Kaur, "Review on text classification by NLP approaches with machine learning and data mining approaches," IJARIIT Journal, vol. 3, no. 4, pp. 767-771, 2017.

[3] S. Parameswaran and D. Joseph, "A review of machine learning techniques used for video classification," IJCESR Journal, vol. 4, no. 12, pp. 64-69, 2017.

[4] J. Pennington, R. Socher, and C. Manning, "Glove: Global vectors for word representation," Proceedings of the 2014 Conference on Empirical Methods in Natural Language Processing (EMNLP), pp. 1532-1543, Doha, Qatar, 2014.

[5] T. Mikolov, K. Chen, G. Corrado, and J. Dean, "Efficient estimation of word representations in vector space," Proceedings of the International Conference on Learning Representations, January 2013.

[6] P. Bojanowski, E. Grave, A. Joulin, and T. Mikolov, "Enriching word vectors with subword information," arXiv preprint arXiv:1607.04606 [Online]. Available: https://research.fb.com/fasttext, 2016.

[7] B. Cui, C. Zhang, and G. Cong, "Content-enriched classifier for Web video classification," Proceedings of the 33rd International ACM SIGIR Conference on Research and Development in Information Retrieval, pp. 619-626, USA, 2010.

[8] S. Schmiedeke, P. Xu, I. Ferrané, M. Eskevich, C. Kofler, M. Larson, Y. Estève, L. Lamel, G. Jones, and T. Sikora, "Blip10000: A social video dataset containing SPUG content for tagging and retrieval," $A C M$ Multimedia Systems Conference, Oslo, Norway, 2013.

[9] L. Yang, J. Liu, X. Yang, and X.-S. Hua, "Multi-modality web video categorization," Proceedings of the International Workshop on Workshop on Multimedia Information Retrieval, pp. 265-274, NY, USA, September 2007.

[10] J. R. Zhang, Y. Song, and T. Leung, "Improving video classification via YouTube video co-watch data," Proceedings of the 2011 ACM workshop on Social and behavioral networked media access - SBNMA '11, pp. 21-26, Arizona, USA, December 2011.

[11] W.-H. Lin, and A. Hauptmann, "News video classification using SVMbased multimodal classifiers and combination strategies," Proceedings of the tenth ACM international conference on Multimedia, pp. 323-326, NY, USA, December 2002.

[12] "Related Words - Find Words Related to Another Word," [Online]. Available: https://relatedwords.org/.

[13] B. Ionescu, I. Mironica, K. Seyerlehner, P. Knees, J. Schluter, M. Schedl, C. Horia, A. Buzo, and P. Lambert, “ARF @ MediaEval 2012: Multimodal Video Classification," Proceedings of the MediaEval 2012 Workshop, Pisa, Italy.

[14] Semela Tomas, Tapaswi Makarand, Ekenel Hazim Kemal, and Stiefelhagen Rainer, "KIT @ MediaEval 2012: Content-based Genre Classification using Visual Cues," Proceedings of the MediaEval 2012 Workshop, Pisa, Italy, 2012.

[15] S. Schmiedeke, P. Kelm, and T. Sikora, "TUB @ MediaEval 2012 Tagging Task: Feature Selection Methods for Bag-of-(visual)-Words Approaches," Proceedings of the MediaEval 2012 Workshop, Pisa, Italy, 2012.

[16] Y. Shi, M. Larson, P. Wiggers, and C. Jonker, "MediaEval 2012 Tagging Task: Prediction based on One Best List and Confusion Networks," Proceedings of the MediaEval 2012 Workshop, Pisa, Italy, 2012.

[17] P. Xu, Y. Shi, and M. Larson, “TUD @ MediaEval 2012 Genre Tagging Task: Multi-modality Video Categorization with one-vs-all Classifiers," Proceedings of the MediaEval 2012 Workshop, Pisa, Italy, 2012.

[18] J. Almeida, T. Salles, E. Martins, O. Penatti, R. Torres, M. Goncalves, and J. Almeida, "UNICAMP-UFMG @ MediaEval 2012: Genre Tagging Task," Proceedings of the MediaEval 2012 Workshop, Pisa, Italy, 2012. 


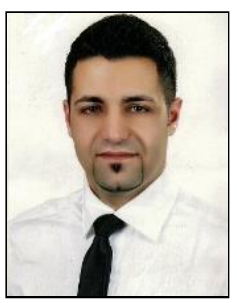

Zein Al Abidin Ibrahim Ph.D. in Computer Science (Image, Information, Hypermedia) from Toulouse University in France, Master and B.S. in Computer Science from the Lebanese University, Faculty of Science, section 1, Beirut, Lebanon. He occupied a postdoc position at the INRIA-IRISA institute of research of Rennes for 16 months to work on

TV stream structuring and a research engineer position at the IRIT institute of research for one year to work on the automatic and the hierarchical video classification for an interactive platform of enhanced digital Television in France. He filled also the position of assistant professor for one year at Caen University and for one year at Anger University in France. Since 2012, he is an associate professor at the Lebanese University - Faculty of Science, Beirut, Lebanon. Among his topics of interest are multimedia content analysis and machine learning especially video and TV streams representation, structuring, comparison and clustering.

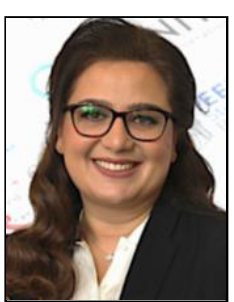

Siba Haidar defended a Ph.D. Thesis in Computer Science (Multimedia) on "Audiovisual document comparison using similarity matrix" in 2005 at the Paul Sabatier University, Toulouse, France. Before that, she received her MSc degree in informatics from the Lebanese University in 2000. Since 2005, she joined the Faculty of Sciences, Lebanese University, where she is currently an associate professor in the department of Computer Science. Her research interests include video analysis and indexing, surveillance video transcription, machine learning. She is a member of the research team LARICOD.

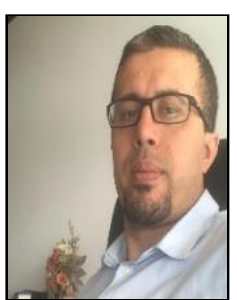

Ihab Sbeity, is a Professor in Computer Science at the Lebanese University. He received a Maîtrise in applied mathematics from the Lebanese university, a Master in computer science - systems and communications from Joseph Fourier university, France, and a $\mathrm{PhD}$ from Institut National Polytechnique de Grenoble (INPG), France. His $\mathrm{PhD}$ works are related to Performance Evaluation and System Design. Currently, Dr. Sbeity occupies a full-time professor position at the Lebanese university - Faculty of Sciences I - computer sciences department. His research interests include software engineering, decision making, and deep learning applications. 